\title{
Comparative Evaluation of Nonprocedural Nephrostomy-Related Complications in Patients with Ureteric Obstruction due to Cancer versus Other Causes: Is the Protocol of Routine 3-month Nephrostomy Exchange Optimal for Both Disease Processes?
}

\author{
Tianshen $\mathrm{Hu}^{1, \dagger}$ Ning Zhang ${ }^{1, \dagger} \quad$ Joseph L. Mcdevitt ${ }^{1} \quad$ Ayobami Odu ${ }^{1} \quad$ Yin $\mathrm{Xi}^{1} \quad$ Daniel Lamus $^{1}$ \\ Patrick Sutphin ${ }^{1}$ Sanjeeva Kalva ${ }^{1}$ Anil K Pillai
}

${ }^{1}$ Department of Radiology, University of Texas Southwestern Medical Center, Dallas, Texas, United States

2Department of Radiology, University of Texas University of Texas Health Science Center at Houston, Houston, Texas, United States

Address for correspondence Tianshen Hu, MD, University of Texas Southwestern Medical Center, 5323 Harry Hines Blvd, Dallas, TX 75390,, United States (e-mail: tianshen.hu@phhs.org).

J Clin Interv Radiol ISVIR 2018;2:23-26

\begin{abstract}
Keywords

- nephrostomy tube exchange

- malignant ureteral obstruction

- benign ureteral obstruction

Purpose To evaluate nonprocedural percutaneous nephrostomy (PCN) complications and assess whether the protocol of routine 3-month exchange of PCN is optimal in patients with malignant ureteral obstruction (MUO) and benign ureteral obstruction (BUO). Materials and Methods Retrospective study of 103 patients with a mean age of 48 years (36 men, 67 women) who underwent PCN placement and subsequent PCN exchanges between January 2011 and January 2014 at the institution was conducted. Comparisons of the number of catheter days for those with routine exchange and emergency exchange due to tube obstruction, infection, and mechanical failures were made in patients with $\mathrm{MUO}$ and $\mathrm{BUO}$.

Results Of the 256 PCN exchange procedures, 139 were performed in the cancer cohort and 117 in the noncancer cohort. Complications requiring emergent exchanges were more common in cancer patients, particularly due to obstruction, which accounted for $57 \%$ of exchanges in MUO with a median onset of 50 days. In contrast, the majority of noncancer patients in this study did not require emergent exchange before 3 months. Routine exchanges accounted for $43 \%$ and $55 \%$ of exchanges in MUO and BUO, respectively. In both the groups, only a small proportion of emergent tube exchanges occurred beyond 105 days.

Conclusion A routine 3-month nephrostomy exchange protocol may not be ideal for all patients. The optimal timing for exchange of nephrostomy appears to be earlier for patients with MUO compared with those with BUO. A prospective study is required to define an optimal policy for postprocedural nephrostomy tube care in both the cohorts.
\end{abstract}

\section{Introduction}

The insertion of a percutaneous nephrostomy (PCN) catheter was first described by Goodwin et al in 1955, and it is a common procedure performed by the interventional radiologists. ${ }^{1}$ The collecting system of the kidney is accessed through the overlying flank, and it provides a temporary or

${ }^{\dagger}$ Contributed equally.

received

March 15, 2017

accepted after revision

December 4, 2017
DOI https://doi.org/

$10.1055 / \mathrm{s}-0038-1641674$

ISSN 2457-0214.
Copyright $\odot 2018$ by Indian Society of Vascular and Interventional Radiology
License terms

() (1) $\Theta \circledast$ 
permanent alternative drainage of urine. The most common indication for PCN is urinary obstruction. Nonobstructive conditions including urinary fistulae, ureteric injury requiring urinary diversion, and percutaneous access to perform nephrolithotomy are less frequent indications for PCN. The technical aspects and the periprocedural care of PCN catheters have been well studied. ${ }^{2-5}$ However, there are no evidence-based guidelines for long-term management of these catheters after placement, as literature regarding late complications is scarce. ${ }^{6}$ The recommended time frame for PCN exchange ranges from 1 to 6 months, based on institutional preferences. At the institution, PCN exchanges are performed every 3 months irrespective of the underlying disease process. Cancer patients are at an overall higher risk of developing complication compared with noncancer patients. ${ }^{7}$ The incidence of PCN catheter-related complications has been reported to be as high as $19 \%$ in cancer patients. ${ }^{7.8}$ The objective of this study is to determine the timeline of nephrostomy exchanges in both cancer and noncancer patients and to determine whether and at what interval a routine exchange would be recommended.

\section{Materials and Methods}

\section{Study Population}

Institutional review board (IRB) approval for this study was obtained in October 2014 from University of Texas Southwestern Medical Center, Dallas, Texas, United States, and this study is in compliance with Health Insurance Portability and Accountability Act (HIPAA) guidelines for research. All patients who underwent $\mathrm{PCN}$ placement/exchange with at least one subsequent follow-up PCN exchange between January 2011 and January 2014 were identified from the hospital database. Patients who had placement of a PCN during the study period with no follow-up exchanges, procedures involving other percutaneous ureteric catheters (e.g., double-J stents, nephroureteral stents), and PCN exchanges performed within 48 hours of placement/exchange were excluded, as these are typically due to periprocedural complications.

\section{Data Review}

Data of the electronic medical records about the patients who underwent PCN placement/exchange procedures were reviewed and recorded into a database that included date and reason for PCN placement/exchange. An exchange was defined as routine or elective if the patient presented for the scheduled PCN exchange at $90 \pm 15$ days and did not have complication at the time of exchange; that is, the patient presented with a normal functioning PCN without obstruction, infection, or mechanical failure of the tube. An exchange was defined as emergent or nonelective if the patient presented secondary to infection, tube obstruction, or mechanical failure. Infections were considered to be present if the patient presented with pyuria, flank pain, fever, or sepsis associated with elevated white blood cell count or positive urine/blood cultures. Asymptomatic bacteria and catheter exit site infections were excluded. Mechanical
Table 1 Patient characteristics $(n=103)$

\begin{tabular}{|c|c|}
\hline Characteristics & Value \\
\hline Mean age (y) & 48.35 \\
\hline Men/women & $36 / 67$ \\
\hline \multicolumn{2}{|l|}{ Disease process: } \\
\hline Cancer & 48 \\
\hline Bladder & 4 \\
\hline Cervical & 22 \\
\hline Colorectal & 7 \\
\hline Prostate & 5 \\
\hline Others/metastasis & 10 \\
\hline Nephrolithiasis & 39 \\
\hline
\end{tabular}

failure included catheter dislodgement (partial or complete), malposition, or catheter defects (catheter being cut). Catheter obstruction was considered to be present if there was no output from the PCN without signs of infection or evidence of mechanical failure. Catheter survival day was defined as the time in days between the date of the exchange of interest and the date of the most recent previous placement or exchange (e.g., if a certain exchange was the patient's third complication during the study period, the time was determined from the date of the second complication rather than the date of the initial placement). Other characteristics recorded in the database included sex, age, race, reason for initial placement (e.g., cancer, nephrolithiasis, and other less common reasons for PCN such as retroperitoneal fibrosis). - Table 1 shows the distribution of study group characteristics.

\section{Statistical Analyses}

Comparisons of the mean, median, and standard deviation for the number of catheter days for tube exchange were made within each patient group (e.g., routine vs. obstruction, routine vs. infection, routine vs. mechanical, etc.) and between the cancer and noncancer patient groups. Statistical analyses were performed using unpaired Student $t$-test or one-way analysis of variance (ANOVA) followed by post-hoc Student-Newman-Keuls test when applicable. $p$ value $\leq 0.05$ was considered statistically significant.

\section{Results}

\section{Patient Characteristics}

Total 103 patients underwent initial PCN placement followed by one or more PCN exchanges during the study period, accounting for a total of 256 procedures.

Of these, 48 patients had cancer whereas 55 patients did not. Twenty-four (50\%) cancer patients had more than one PCN exchange procedure, whereas $22(40 \%)$ noncancer patients had more than one PCN exchange. The number of patients who had bilateral PCNs were 14 and 11 in the cancer and non-cancer groups, respectively. The mean age of the cancer patients was 53.7 years compared with 43.6 in the noncancer cohort. The cancer cohort had 37 females compared with 30 in the noncancer cohort. 


\section{Reason for Catheter Exchanges:}

Of the 256 PCN exchanges, 139 were performed in the cancer cohort and 117 in the noncancer cohort. Routine exchanges accounted for $43 \%$ of the cases in cancer patients and $55 \%$ in noncancer patients. Nonroutine exchanges were more common $(57 \%$ vs. $45 \%)$ in the cancer patients compared with the noncancer cohort, and they accounted for more than half of the procedures in cancer patients. Among the complications, obstruction was the most common cause in cancer patients, which accounted for $32 \%$ of total procedures, followed by infection (16\%) and mechanical failure (9\%). In the noncancer group, the number of cases of obstruction, infection, and mechanical failures is very similar, accounting for 17 to $18 \%$ of emergent tube exchange procedures.

\section{Catheter Survival Duration}

Catheter survival days for routine and nonroutine PCN exchanges are recorded in - Table 2. Comparing the routine exchanges for the cancer and noncancer cohort showed that the catheters were exchanged at $93 \pm 21$ and $102 \pm 23$ days, respectively (not statistically significant). The catheter survival days in the cancer cohort were $50 \pm 32$ days for obstruction, $96 \pm 50$ days for infection, and $55 \pm 23$ days for mechanical failure. The catheter survival days were $103 \pm 81,93$ \pm 48 , and $65 \pm 50$ days for obstruction, infection, and mechanical failures, respectively, in the noncancer cohort. A statistically significant difference was observed when comparing the catheter survival days in the cancer versus the noncancer cohort only in the PCN obstruction category $(p<0.05)$. In the cancer cohort, $56 \%$ of the nonroutine exchanges were due to obstruction $(n=45)$. In comparison, there was not a predominant reason for nonroutine exchange in noncancer patients ( $n=17$ for obstruction, $n=18$ for infection and mechanical). Mechanical issues in both patient groups tend to occur earlier than 3 months and have much shorter catheter survival days.

- Fig. 1 is the distribution of frequency of complications occurring in the cancer and noncancer cohorts. In the cancer cohort, more than half of those who had obstruction experienced it within 45 days of tube insertion (median of 39 days), and the rate of mechanical failure was highest between 45 and 76 days (median of 51 days). In contrast, $64 \%$ of noncancer patients in this study did not require a nonroutine tube

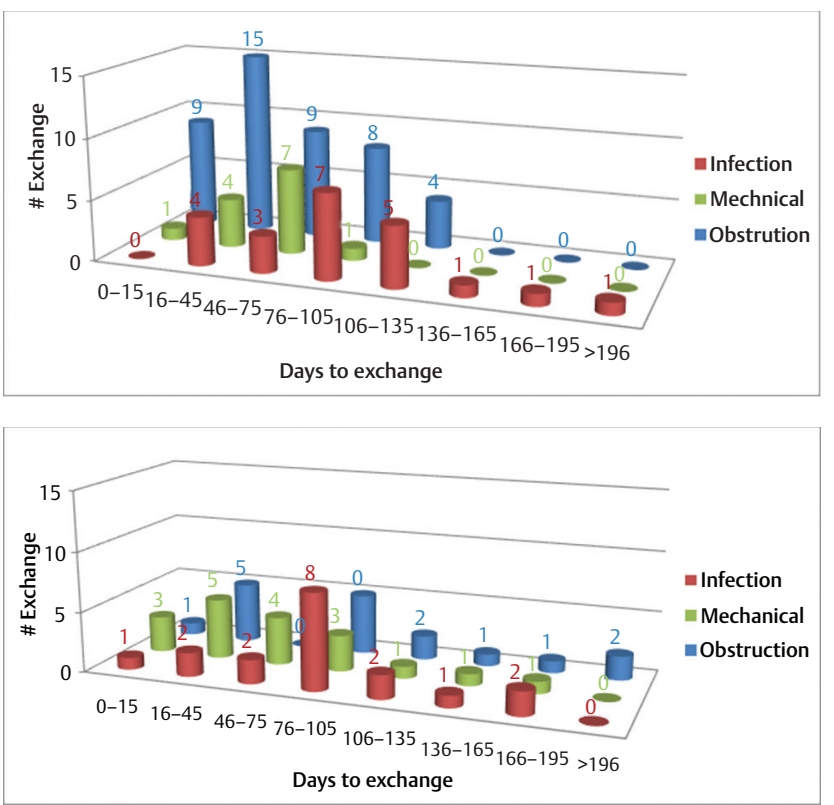

Fig. 1 (A) Comparison of causes of PCN failure in patients with cancer. Obstruction was observed to have an early peak between 16 and 45 days. Mechanical failures also tend to occur before the 3-month routine exchange. The highest rate of infection occurred during 76 to 105 days. (B) Comparison of causes of tube failure in patients without cancer. The complication risks were less common and relatively more evenly spread in this group.

exchange before 3 months. The leading cause of early nonroutine tube exchange in this patient population is mechanical failure such as due to tube dislodgment, malposition, or leakage. In both groups, only a small proportion of emergent tube exchanges occurred beyond 105 days.

\section{Discussion}

PCN placement has an overall technical success of 88 to $94 \%$ for urinary decompression with approximately $10 \%$ major and minor complication rate combined and $0.2 \%$ mortality rate. ${ }^{9,10}$ Postprocedural management of PCN is not standardized, and there are limited data on the optimal time for exchanges. ${ }^{11,12}$ The clinical presentation, definitive treatment, and outcomes are different for malignant and benign ureteral strictures requiring a PCN. ${ }^{11}$ Benign

Table 2 Catheter days until PCN exchange

\begin{tabular}{|l|l|l|l|l|l|}
\hline \multicolumn{2}{|c}{ Routine } & \multicolumn{2}{l}{ Obstruction } & \multicolumn{2}{l|}{ Infection } \\
\hline \multirow{3}{*}{ Cancer } & Mean \pm SD & $102 \pm 23$ & $50 \pm 32^{\mathrm{a}, \mathrm{b}}$ & $96 \pm 50^{\mathrm{c}}$ & $55 \pm 23^{\mathrm{b}, \mathrm{d}}$ \\
\cline { 2 - 6 } & Median, 25\% & 96,90 & 39,24 & 90,59 & 51,42 \\
\cline { 2 - 6 } & $\mathrm{N}$ & 59 & 45 & 22 & 13 \\
\hline \multirow{3}{*}{ Noncancer } & Mean \pm SD & $93 \pm 21$ & $103 \pm 81$ & $93 \pm 48$ & $65 \pm 50^{\mathrm{b}, \mathrm{d}, \mathrm{d}}$ \\
\cline { 2 - 6 } & Median, 25\% & 91,90 & 86,35 & 91,73 & 53,24 \\
\cline { 2 - 6 } & $\mathrm{N}$ & 64 & 17 & 18 & 18 \\
\hline
\end{tabular}

Abbreviations: PCN, percutaneous nephrostomy; SD, standard deviation.

${ }^{a} p<0.01$ between cancer versus noncancer in obstruction group.

${ }^{b} p<0.05$ versus the respective routine group.

${ }^{c} p<0.05$ versus the respective obstruction group.

${ }^{\mathrm{d}} p<0.05$ versus the respective infection group. 
ureteral obstruction (BUO) typically presents acutely with pain, nausea, and vomiting associated with or without signs of pyelonephritis whereas malignant ureteral obstruction (MUO) is often asymptomatic or may present with uremia or vague abdominal discomfort. Rarely MUO presents acutely with pyelonephritis/sepsis. The indication for urinary decompression is for temporary relief before definitive treatment in BUO whereas PCN placement is more likely to be a definitive measure to preserve renal function in MUO. Patients with MUO usually have a poor prognosis and lower overall survival with limited chance for complete cure unlike BUO. ${ }^{11}$

This study showed higher complication rates necessitating earlier PCN exchange in cancer patients compared with noncancer patients. The reasons for high PCN failure rate early on in cancer patients have not been studied. Monsky et al also reported a higher rate of complications, including catheter dislodgments, pain, infection, and clogging in patients receiving PCN for MUO. In their study, $83 \%$ required additional PCN changes within the 3-month period. ${ }^{12}$ Bahu et al reported only $45 \%$ of cancer patients underwent routine catheter exchange per the institutional policy. ${ }^{7}$ In their cohort, the most common complication was tube obstruction (24\%) followed by pyelonephritis (19\%), within 3 months of the procedure, whereas half of those cases occurred within the first month. Their findings are similar to ours in which routine exchanges were done only in $43 \%$ of the exchanges and catheter obstruction was the most common complication accounting for $32 \%$ of all exchanges and infection $16 \%$.

In contrast, $55 \%$ of patients with BUO underwent routine PCN exchange. Compared with MUO, patients with BUO are relatively younger with better performance status. At the institution, patients are referred for a routine exchange by noninterventional radiology (non-IR) clinics. Although the institutional policy is for a routine exchange at 3 months, this is not strictly adhered to due to variability of clinic follow-up. These complications are preventable with a strict follow-up program. The authors have initiated an IR-driven PCN clinic to address this nonuniformity.

There are limitations to this study. The procedural indication, patient education regarding tube hygiene, the method, and timing of follow-up are not consistently documented. Patients are referred for PCN exchanges from different specialties with variable follow-up policies. Many of the patients have routine PCN exchanges and complications beyond the 3-month mark, which would bias the data. This study excluded all patients who returned for PCN-related complications within the first 48 hours as the authors defined these as procedure related. Though the authors are interested in nonprocedural-related complications, they are fully aware that the 48-hour time limit is arbitrary, and that there is no strict timeline to separate procedure- versus nonprocedural-related complications. Last, for those with bilateral PCN, exchanges are typically performed for both PCNs at the same time, and they were considered two procedures in this analysis. In conclusion, patients who receive PCN for BUO and MUO are different and require different follow-up protocols. Patients with MUO develop PCN tube-related complications earlier than those with BUO. The complication rates are higher in MUO. A universal 3-month nephrostomy tube exchange policy may not be ideal for both the groups. A prospective study with an established IR-driven PCN clinic is required to define an optimal policy for postprocedural nephrostomy tube care in both the cohorts.

\section{Conflict of Interest}

None.

\section{References}

1 Goodwin WE, Casey WC, Woolf W. Percutaneous trocar (needle) nephrostomy in hydronephrosis. J Am Med Assoc 1955;157(11):891-894

2 Patel U, Hussain FF. Percutaneous nephrostomy of nondilated renal collecting systems with fluoroscopic guidance: technique and results. Radiology 2004;233(1):226-233

3 Farrell TA, Hicks ME. A review of radiologically guided percutaneous nephrostomies in 303 patients. J Vasc Interv Radiol 1997;8(5):769-774

4 Hausegger KA, Portugaller HR. Percutaneous nephrostomy and antegrade ureteral stenting: technique-indications-complications. Eur Radiol 2006;16(9):2016-2030

5 Zagoria RJ, Dyer RB. Do's and don't's of percutaneous nephrostomy. Acad Radiol 1999;6(6):370-377

6 Nephrostomy and Biliary Tube Management. A Review of the Clinical Evidence and Guidelines. Ottawa, ON, Canada: CADTH Rapid Response Reports; 2014

7 Bahu R, Chaftari AM, Hachem RY, et al. Nephrostomy tube related pyelonephritis in patients with cancer: epidemiology, infection rate and risk factors. J Urol 2013;189(1):130-135

8 Plesinac-Karapandzic V, Masulovic D, Markovic B, et al. Percutaneous nephrostomy in the management of advanced and terminal-stage gynecologic malignancies: outcome and complications. Eur J Gynaecol Oncol 2010;31(6):645-650

9 Lee WJ, Patel U, Patel S, Pillari GP. Emergency percutaneous nephrostomy: results and complications. J Vasc Interv Radiol 1994;5(1):135-139

10 Stables DP. Percutaneous nephrostomy: techniques, indications, and results. Urol Clin North Am 1982;9(1):15-29

11 Hsu L, Li H, Pucheril D, et al. Use of percutaneous nephrostomy and ureteral stenting in management of ureteral obstruction. World J Nephrol 2016;5(2):172-181

12 Monsky WL, Molloy C, Jin B, et al. Quality-of-life assessment after palliative interventions to manage malignant ureteral obstruction. Cardiovasc Intervent Radiol 2013;36(5):1355-1363 